\title{
Rapid and Sensitive Quantification of the Pesticide Lindane by Polymer Modified Electrochemical Sensor
}

\author{
Jafar Safaa Noori $^{1, * \mathbb{D}}$, John Mortensen ${ }^{2}$ and Alemnew Geto ${ }^{1}$ D \\ 1 IPM—Intelligent Pollutant Monitoring ApS, 2690 Karlslunde, Denmark; alg@intpm.dk \\ 2 Department of Science and Environment, Roskilde University, 4000 Roskilde, Denmark; john@ruc.dk \\ * Correspondence: jasnoo@intpm.dk
}

check for updates

Citation: Noori, J.S.; Mortensen, J.; Geto, A. Rapid and Sensitive Quantification of the Pesticide Lindane by Polymer Modified Electrochemical Sensor. Sensors 2021, 21, 393. https://doi.org/10.3390/ s21020393

Received: 21 December 2020 Accepted: 6 January 2021 Published: 8 January 2021

Publisher's Note: MDPI stays neutral with regard to jurisdictional clai$\mathrm{ms}$ in published maps and institutional affiliations.

Copyright: (C) 2021 by the authors. Licensee MDPI, Basel, Switzerland. This article is an open access article distributed under the terms and conditions of the Creative Commons Attribution (CC BY) license (https:// creativecommons.org/licenses/by/ $4.0 /)$.

\begin{abstract}
Lindane is documented by the Environmental Protection Agency (EPA) as one of the most toxic registered pesticides. Conventional detection of lindane in the environment requires manual field sampling and complex, time-consuming analytical sample handling relying on skilled labor. In this study, an electrochemical sensing system based on a modified electrode is reported. The system is capable of detecting lindane in aqueous medium in only $20 \mathrm{~s}$. The surface of a conventional carbon electrode is modified with a film of conductive polymer that enables detection of lindane down to 30 nanomolar. The electrode modification procedure is simple and results in a robust sensor that can withstand intensive use. The sensitivity of the sensor is $7.18 \mu \mathrm{A} / \mu \mathrm{M}$ and the performance was demonstrated in the determination of lindane in spiked ground water. This suggests that the sensor is potentially capable of providing useful readings for decision makers. The rapid and sensitive quantification of lindane in aqueous medium is one step forward to new opportunities for direct, autonomous control of the pesticide level in the environment.
\end{abstract}

Keywords: pesticide; lindane; polymer; electrochemical; sensor; water; contamination

\section{Introduction}

Lindane $(1 \alpha, 2 \alpha, 3 \beta, 4 \alpha, 5 \alpha, 6 \beta$-hexachlorocyclohexane) is a polychlorinated insecticide used in agriculture to protect fruits, vegetables and crops from insects, eggs and larvae [1]. Lindane has the potential to significantly alter ecosystems, pose toxicity to animals and humans and be accumulated in the food chain. The heavy use of lindane in agriculture has led to contamination of the environment [2]. Exposure to lindane may occur from eating contaminated food or by drinking contaminated water [3]. Intake of lindane is associated with neurotoxic effects and may cause anemia and significant damage to the liver, and the immune system [4].

Currently, various analytical methods are available for the detection and quantification of lindane in the environment [5]. Common for most is that they involve collection of samples, retrieval of the samples to a central laboratory and labor-intensive sample clean-up and pretreatment steps followed by quantification [6]. In addition to the lengthy and uneconomical steps involved, degradation of lindane may occur during transportation of the samples from the field causing inaccurate analytical results [7]. Thus, it is desirable to develop techniques that enable rapid in situ measurements in the environment. Electrochemical sensors present a promising alternative to conventional determination of lindane in the environment due to its rapid and sensitive measurement protocol. Furthermore, recent advances in electronics has enabled electrochemical sensors to have the potential to be employed for onsite detection $[1,8,9]$.

The first step towards onsite electrochemical detection of lindane in the environment is to develop selective detection of the compound. However, only few reports are available in the literature regarding the electrochemistry of lindane in conventional and modified electrodes. Earlier reports focused on the reduction of the compound on mercury based electrodes [10] which is currently a concern of health and environmental pollution itself. 
Other electrochemical studies of lindane on modified and non-modified electrodes were available either in a completely organic media [11-14] or aqueous-organic mixtures [15-18] making them less attractive for direct environmental application. Moreover, the peak potential for the reduction of lindane was reported to occur at $-1.45 \mathrm{~V}[18],-1.5 \mathrm{~V}[15,17,19]$ $-1.94 \mathrm{~V}$ (vs. $\mathrm{Ag} / \mathrm{AgCl}$ ) [11], or -1.40 and $-2.10 \mathrm{~V}[12,14]$, which is highly negative for selective determination due to interference problems from co-existing compounds. A study [16], on the reduction of lindane at silver cathodes in organic and aqueous-organic media showed a shift of the reduction peak to less negative potential with the addition of water from $-1.40 \mathrm{~V}$ in $100 \%$ acetonitrile $(\mathrm{ACN})$ to $-0.89 \mathrm{~V}$ in $50: 50 \mathrm{ACN}-\mathrm{H}_{2} \mathrm{O}$. This shows the significant influence of the medium in the reduction potential of lindane shifting positively with increasing the water proportion. Therefore, the objective of this study is to develop an electrochemical method for the determination of lindane in a completely aqueous medium using a polymer modified carbon electrode.

The polymer film used in this study was electrochemically synthesized from 4-amino3-hydroxynaphtahele sulfonic acid (4A3HNSA) in $0.1 \mathrm{HNO}_{3}$. The polymer deposition process and the property of the resulting film was characterized using cyclic voltammetry $(\mathrm{CV})$, scanning electron microscopy(SEM) and electrochemical impedance spectroscopy(EIS) [20] and it was found to result in remarkably robust electrodes. The polymer film modified glassy carbon electrode has been reported previously for the determination of various bioactive molecules [21-23]. However, all previous uses of the electrode were limited on the oxidation of the compounds and the current report is the first attempt to apply it in the reductive detection.

\section{Experimental}

\subsection{Reagents and Solutions}

Chemicals and reagents used in this study were all analytical grade and used as received without any pretreatment. Aqueous solutions were prepared in Milli-Q water (resistivity $\geq 18 \mathrm{M} \Omega \mathrm{cm})$. Lindane $(1 \alpha, 2 \alpha, 3 \beta, 4 \alpha, 5 \alpha, 6 \beta$-hexachlorocyclohexane), 4amino-3-hydroxynaphthalene sulfonic acid, chlorobenzene, 4-chlorobenzaldehyde, 1,3,5trichlorobenze and $N, N$-Dimethylformamide (DMF) were all supplied by Sigma-Aldrich. $\mathrm{KCl}$ was purchased from Frederiksen Scientific A/S. $10 \mathrm{mM}$ stock solution was prepared by dissolving lindane in $\mathrm{N}, \mathrm{N}$-Dimethylformamide (DMF) and kept in brown bottle in the fridge. All working solutions were prepared by diluting appropriate volume of the stock solution in Milli-Q water containing supporting electrolyte.

Ground water sample was analyzed by spiking different concentrations of lindane. The water sample was collected from Gevninge waterworks, Southern Copenhagen directly after pumping it out from the well. Until analysis, the sample was stored in a tight container to avoid oxygen contact and kept in the dark at $4{ }^{\circ} \mathrm{C}$.

\subsection{Experimental Setup}

All electrochemical measurements were conducted in a conventional three electrode setup as seen in Figure 1. The electrochemical cell consists of bare or polymer modified glassy carbon electrode ( $3 \mathrm{~mm}$ diameter) as a working electrode, Pt wire as counter electrode and $\mathrm{Ag} / \mathrm{AgCl}$ reference electrode. The electrodes were connected to a potentiostat (EmStat 3+, PalmSense, The Netherlands) and a computer system from which the applied settings were controlled. All data analyses were conducted with PSTrace 5.5 (PalmSense, Houten, The Netherlands). 


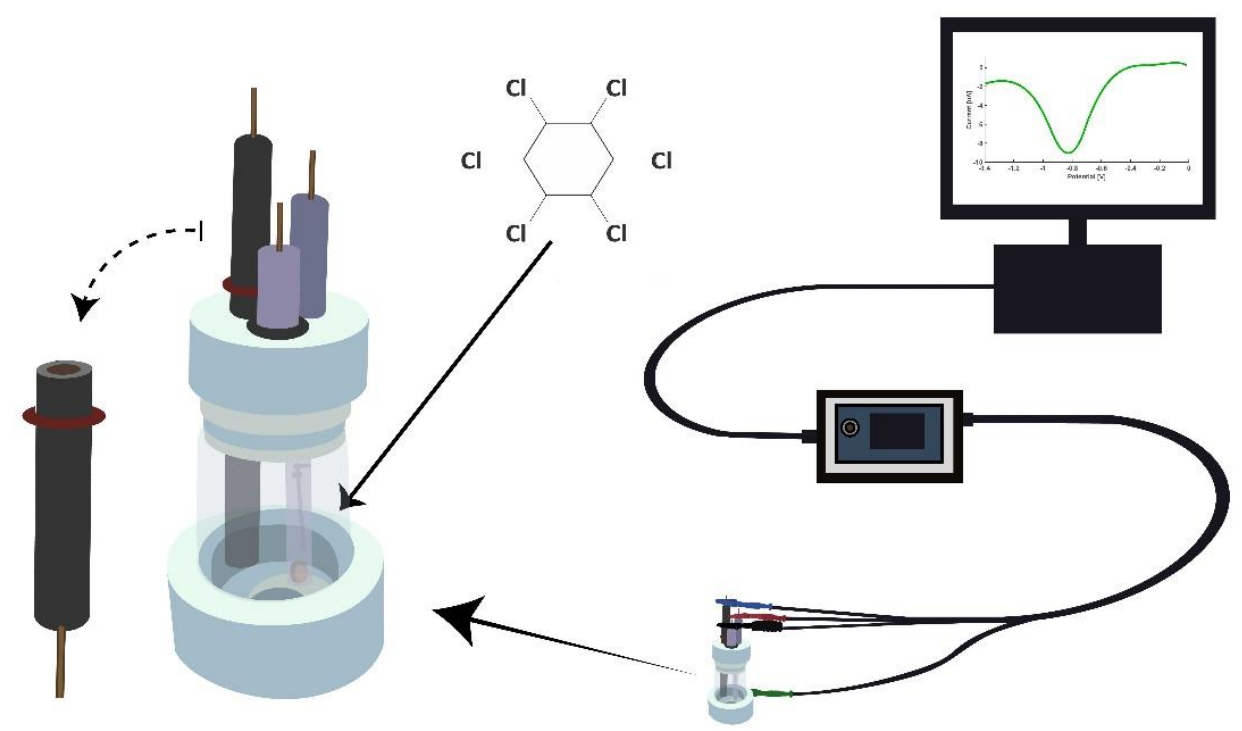

Figure 1. Electrochemical setup used in the study consisting of electrochemical cell, a potentiostat connected to a computer system to run the measurement and record data.

\subsection{Preparation of the Working Electrode}

Prior to use, glassy carbon electrode was polished with 0.3 and $0.05 \mu \mathrm{m}$ alumina slurry on a polishing pad and rinsed thoroughly with Milli-Q water. The clean electrode was placed in $2 \mathrm{mM}$ monomer solution which was prepared by dissolving 4-amino-3hydroxynaphthalene sulfonic acid in $0.1 \mathrm{M} \mathrm{HNO}_{3}$. Electrochemical polymerization was conducted by scanning the potential between $-0.8 \mathrm{~V}$ and $+2.0 \mathrm{~V}$ using cyclic voltammetry for 15 cycles at $0.1 \mathrm{~V} \mathrm{~s}^{-1}$, a thin film of the conducting polymer is formed at the electrode surface. Cyclic voltammetry scans between $-0.8 \mathrm{~V}$ and $+0.8 \mathrm{~V}$ in $0.5 \mathrm{M} \mathrm{H}_{2} \mathrm{SO}_{4}$ was conducted to stabilize the film and to remove any non-adsorbed items from the electrode surface. Further details about the film formation and characterization can be found in a previous report [20].

\subsection{Electrochemical Measurements}

Cyclic voltammetry (CV) and square wave voltammetry (SWV) were used to study the electrochemical behavior of lindane. In the studies, $10 \mathrm{~mL}$ of $0.2 \mathrm{M} \mathrm{KCl}$ solution was placed in the electrochemical cell to record the background response and appropriate volume of $10 \mathrm{mM}$ lindane in DMF was added to it and mixed thoroughly to record voltammograms of the compound. SWV was used to study the electrochemical behavior in the absence and presence of $100 \mu \mathrm{M}$ lindane at bare and polymer modified glassy carbon electrode. The effect of scan rate was studied by running CV measurements in $100 \mu \mathrm{M}$ lindane solution between 40 and $400 \mathrm{mV} \mathrm{s}^{-1}$ in the potential window from 0.0 to $-2.0 \mathrm{~V}$. The interference study was also investigated using SWV in the absence and presence of equal amount of interfering compound in $1 \mu \mathrm{M}$ lindane solution.

In spiked sample analysis, $10 \mathrm{~mL}$ of 20:80 ground water: $0.2 \mathrm{M} \mathrm{KCl}$ was placed and background signal recorded. Then, this mixture was spiked with three concentration of lindane systematically chosen at different levels of the standard calibration curve to make $0.1,0.5$ and $1 \mu \mathrm{M}$ and the response was obtained. The current response from the background corrected voltammogram was plotted against lindane concentration.

\section{Results and Discussion}

\subsection{Electrochemical Behavior of Lindane}

The electrochemical profile of lindane was studied by square wave voltammetry at a bare and polymer modified glassy carbon electrode in $0.2 \mathrm{M} \mathrm{KCl}$ solution (Figure 2). At both electrodes, the voltammograms showed two distinct peaks in the region around $-1.60 \mathrm{~V}$ 
and $-0.80 \mathrm{~V}$ in the presence of $100 \mu \mathrm{M}$ lindane (solid lines) while no significant peaks were observed in the absence of lindane (broken lines). The peak around $-1.6 \mathrm{~V}$ is the significant signal which is also consistent with previous reports $[11,12,14,15,17,18]$ regarding the reduction of lindane at various electrodes. This peak is attributed to the transformation of lindane to benzene through electrochemical reduction involving 6-electrons [24]. The second weak peak observed around $-0.8 \mathrm{~V}$ is not reported in previous studies except in one stating the oxygen mediated reduction of lindane facilitated by the reaction of superoxide from oxygen reduction with lindane [11]. However, preliminary studies in nitrogen saturated medium in the absence and presence of lindane (Figure 3) revealed a consistent reduction peak confirming the absence of oxygen involvement in the process. Rather, the complete reduction of lindane to benzene might be involving a series of intermediate reduction steps by breaking a carbon-chlorine bond to form less chlorinated compounds. The reduction of lindane at lower negative potential is also in agreement with a study by Peverly et al., who reported a shift in the reduction potential to more positive values with an increase in the amount of water during aqueous-organic mixture media at a silver electrode [16]. The effective proton donor property of water is thought to promote the conversion of lindane to benzene.
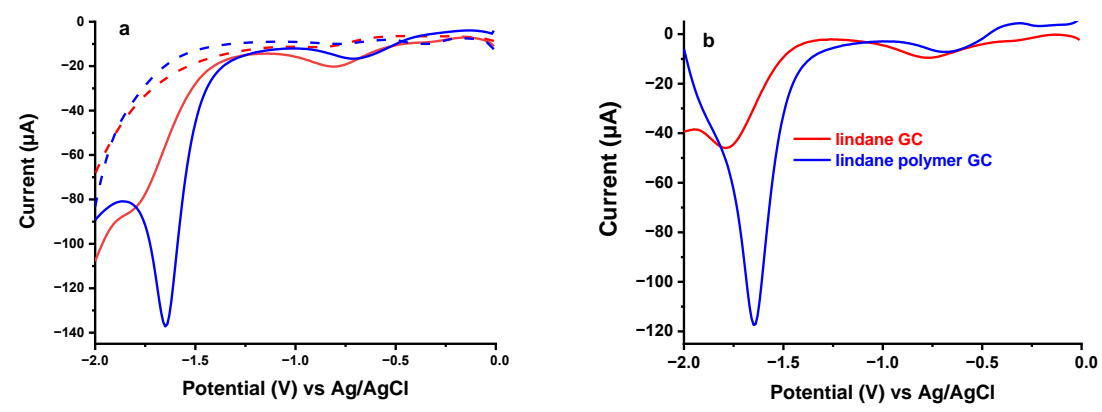

Figure 2. Square wave voltammogram in the absence (broken) and presence (solid) of lindane in $0.2 \mathrm{M}$ $\mathrm{KCl}$ solution at bare (red) and polymer modified (blue) glassy carbon electrode (a) and background corrected response at bare (red) and polymer modified (blue) electrode (b). SWV conditions: $E_{\text {step }}=0.01 \mathrm{~V}$; Amplitude $=0.1 \mathrm{~V}$; Frequency $=15.0 \mathrm{~Hz}$.

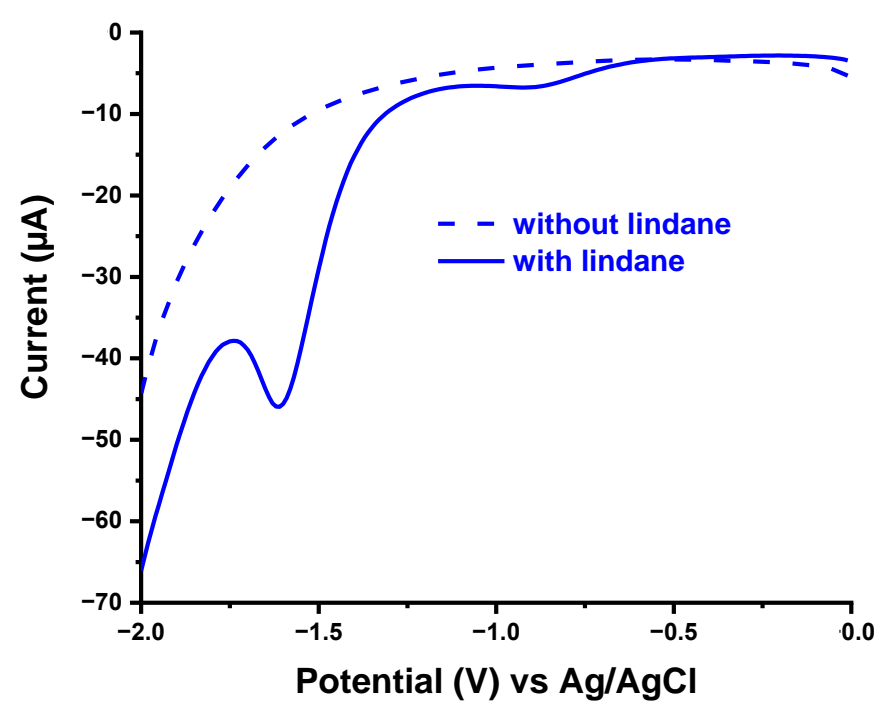

Figure 3. Square wave voltammogram in the absence (broken) and presence (solid) of lindane in nitrogen saturated $0.2 \mathrm{M} \mathrm{KCl}$ solution at glassy carbon electrode. SWV conditions: $\mathrm{E}_{\text {step }}=0.01 \mathrm{~V}$; Amplitude $=0.1 \mathrm{~V}$; Frequency $=15.0 \mathrm{~Hz}$.

Comparing the bare and the polymer modified electrodes, the peak current obtained at the modified electrode is five-times higher than the current recorded at the bare electrode at 
the more negative potential. Whereas, no significant difference in peak response is observed between the electrodes at the less negative region. In addition to this, the reduction peak potentials also shifted positively from $-1.79 \mathrm{~V}$ to $-1.64 \mathrm{~V}$ at high negative region and from $-0.81 \mathrm{~V}$ to $-0.69 \mathrm{~V}$ at lower negative region using the bare and modified electrodes, respectively. This shows the catalytic property of the polymer film on the electrochemical reduction of lindane. The better performance of the polymer modified electrode might be attributed to the presence of $-\mathrm{OH},-\mathrm{SO}_{3} \mathrm{H}$ and $-\mathrm{NH}_{2}$ functional groups in the naphthalenebased polymer film that can contribute to catalytic reduction of lindane. In addition, the naphthalene skeleton of the polymer by itself can better interact with organic and non-polar molecules and could play a role in the better performance. Therefore, the polymer modified electrode is selected for subsequent experiments.

\subsection{Effect of Potential Scan Rate and Buffer $p H$}

Cyclic voltammograms of $100 \mu \mathrm{M}$ lindane were recorded in $0.2 \mathrm{M} \mathrm{KCl}$ solution with different scan rates to investigate the reduction kinetics at the polymer modified electrode (Figure 4). The study was made between 40 and $400 \mathrm{mV} \mathrm{s}^{-1}$ and the peak current at $\sim-1.6 \mathrm{~V}$ was plotted against the scan rate and square root of scan rate, respectively, which the plots yield the linear regression equations: $\mathrm{I}_{\mathrm{p}}(\mu \mathrm{A})=50.14 \nu\left(\mathrm{V} \mathrm{s}^{-1}\right)+9.34\left(\mathrm{R}^{2}=0.996\right)$ and $\mathrm{I}_{\mathrm{p}}(\mu \mathrm{A})=42.19$ $v^{1 / 2}\left(\mathrm{~V}^{1 / 2} \mathrm{~s}^{-1 / 2}\right)+1.35\left(\mathrm{R}^{2}=0.986\right)$. These results depict that the kinetics of lindane reduction at the modified electrode has contributions from both adsorption and diffusion processes [25]. However, the plot of the log peak current $(\mu \mathrm{A})$ against $\log$ scan rate $\left(\mathrm{V} \mathrm{s}^{-1}\right)$ also followed a linear relation according to the equation: $\log \mathrm{I}_{\mathrm{p}}(\mu \mathrm{A})=0.433 \log v\left(\mathrm{~V} \mathrm{~s}^{-1}\right)+1.608\left(\mathrm{R}^{2}=0.978\right)$, with a slope of 0.433 close to 0.5 a theoretical value for a purely diffusion-controlled process, indicating the dominant effect of diffusion in the reduction process [26]. Additionally, a shift in the reduction peak to more negative potential is observed from the plot of $E_{p}$ vs log scan rate following an equation: $E_{p}(V)=0.140 \log v\left(V^{-1}\right)+1.772\left(R^{2}=0.992\right)$ which confirms the complete irreversibility of the reduction process.
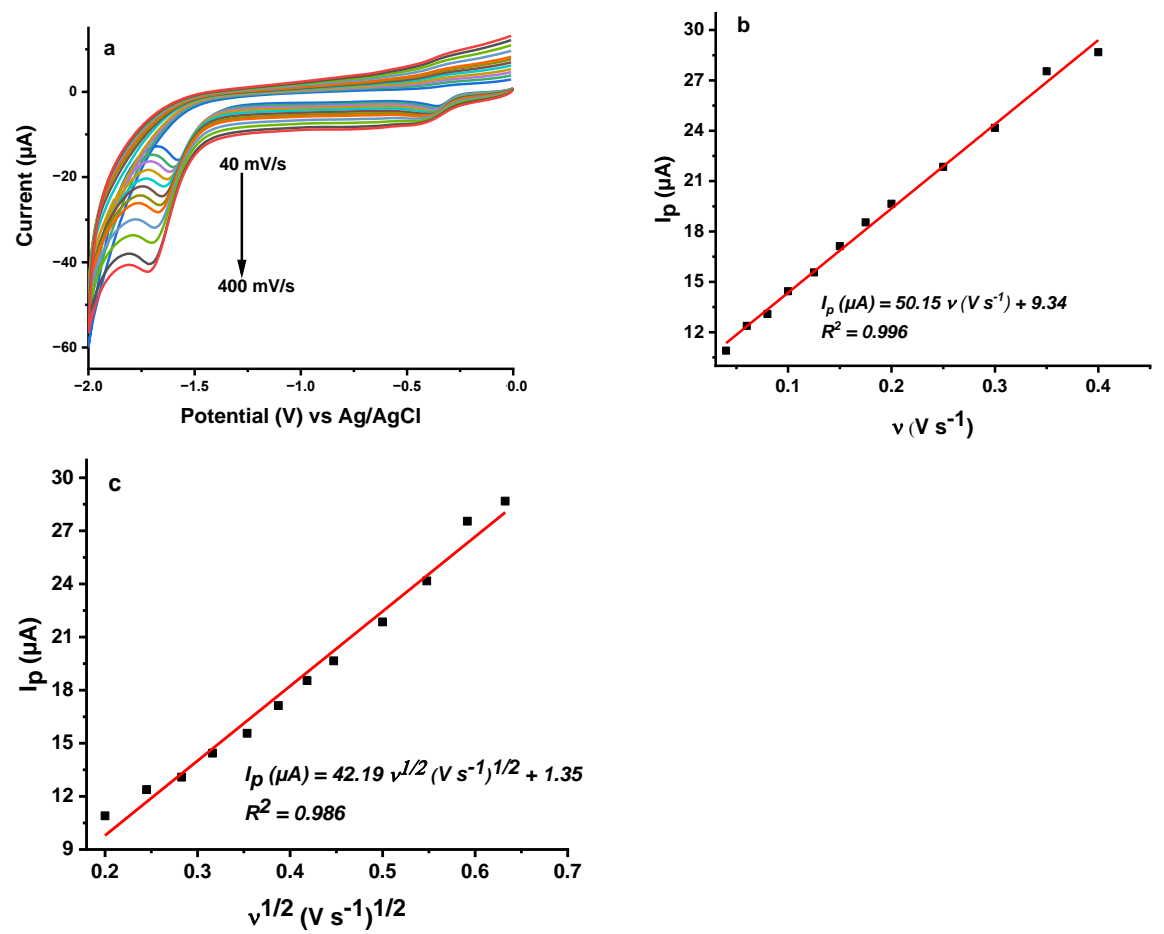

Figure 4. (a) cyclic voltammograms of $100 \mu \mathrm{M}$ lindane using different scan rates in $0.2 \mathrm{M} \mathrm{KCl}$ solution and reduction peak currents extracted from the voltammograms plotted against the scan rates (b) and against the square root of scan rates (c). 
The effect of solution $\mathrm{pH}$ on the reduction of lindane at the polymer modified electrode was investigated in $0.1 \mathrm{M}$ phosphate buffer solution of $\mathrm{pH} 4$ to 9 . Square wave voltammetry was chosen over cyclic voltammetry in this study for better peak resolution and measurements were recorded in $100 \mu \mathrm{M}$ lindane in different $\mathrm{pH}$ conditions. The results did not show a shift in peak potentials with change in $\mathrm{pH}$ which is in agreement with previous reports [10]. However, increasing in buffer $\mathrm{pH}$ led to a decrease in peak currents which might be due to the conductivity loss or film degradation in higher $\mathrm{pH}$ solutions as previously noted in the polymer film [20]. For practical convenience, subsequent experiments were performed in $0.2 \mathrm{M} \mathrm{KCl}$ as supporting electrolyte.

\subsection{Rapid Quantification of Lindane in Aqueous Solution}

The quantification of lindane using the modified electrode was performed using square wave voltammetry. The reduction potential at $\sim-0.8 \mathrm{~V}$ was targeted as the characteristic peak for lindane quantification as it appears at relatively lower negative potential than the strong peak at $\sim-1.6 \mathrm{~V}$. A lower negative potential is preferable to avoid interference from co-existing compounds. Furthermore, preliminary tests show that the peak at $\sim 1.6 \mathrm{~V}$ appears only at higher lindane concentrations which makes the second peak the dominant signal in low concentrations and practically relevant for sensitive determination of lindane.

Square wave voltammograms between 0.0 to $-1.4 \mathrm{~V}$ were recorded to quantify lindane in the concentration range from 0.05 to $1.0 \mu \mathrm{M}$ in $0.2 \mathrm{M} \mathrm{KCl}$ solution (Figure 5a). The background measurement was recorded in $0.2 \mathrm{M} \mathrm{KCl}$ and then a series of lindane standards were spiked to the solution, stirred thoroughly and measurements were performed. The background corrected voltammograms Figure $5 \mathrm{a}$ and the mean peak currents extracted from three independent calibration measurements together with standard deviation used as error bars were plotted (Figure $5 b$ ). Figure $5 b$ shows that the peak current is linearly proportional to the lindane concentration with the sensitivity of $7.18 \mu \mathrm{A} / \mu \mathrm{M}$. The linear regression equation of the plot was: $I_{p}(\mu \mathrm{A})=7.18$ [lindane] $(\mu \mathrm{M})+0.602\left(R^{2}=0.991\right)$.
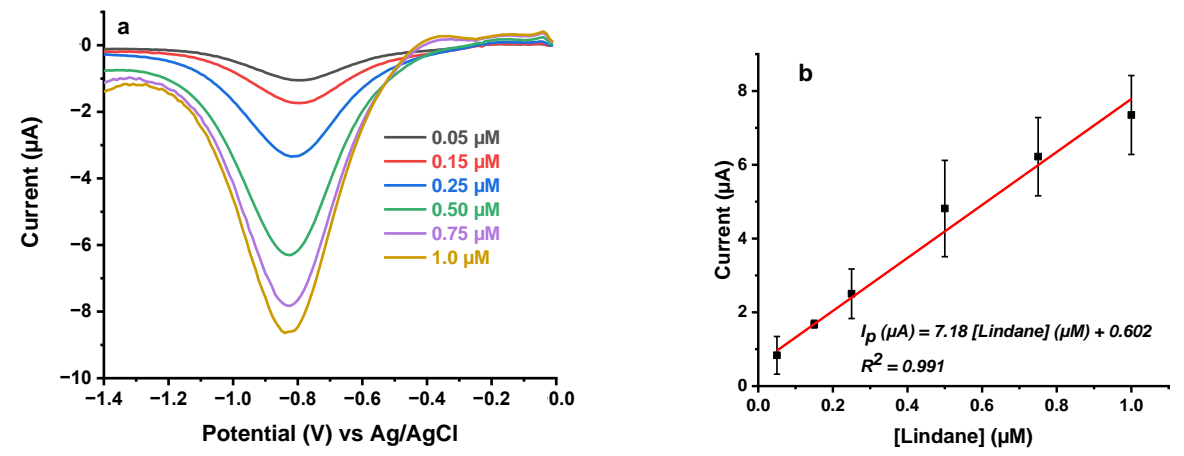

Figure 5. Calibration curve of lindane in $0.2 \mathrm{M} \mathrm{KCl}$ using (a) square wave voltammetry and (b) plot of extracted peak current against lindane concentration. SWV conditions: $E_{\text {step }}=0.01 \mathrm{~V}$; Amplitude $=0.1 \mathrm{~V}$; Frequency $=15.0 \mathrm{~Hz}$.

The theoretical detection and quantitation limits were calculated following the International Conference on Harmonisation (ICH) guideline [27] by $3.3 \times \delta / s$ and $10 \times \delta / s$, respectively; where ' $\delta$ ' is the standard deviation of the y-intercept of the regression line and ' $s$ ' is the slope of calibration curve. Thus, the calculated detection limit (LoD) was found to be $30 \mathrm{nM}$ while the quantitation limit (LoQ) was $98 \mathrm{nM}$. The regulatory limit of pesticides in drinking water is $0.1 \mu \mathrm{g} / \mathrm{L}$ which corresponds to $0.34 \mathrm{nM}$ [28]. This limit of detection is lower than electrochemical methods reported for lindane determination at cellulose acetate/GC [15], $\mathrm{MnO}_{2} / \mathrm{GC}$ [18], $\mathrm{NiCo}_{2} \mathrm{O}_{4} / \mathrm{GC}$ [17] and comparable with vitreous carbon [14], Nylon 6.6/MWCNT/ $\mathrm{Fe}_{3} \mathrm{O}_{4}$ [29] and $\mathrm{CuO}-\mathrm{MnO}_{2} / \mathrm{GC}$ [19]. Moreover, this study is the first attempt to determine lindane in a completely aqueous medium. Summary and comparison of electrochemical methods and their analytical performance in the determination of lindane is shown in Table 1. 
Table 1. Comparison of different electrochemical methods reported for the determination of lindane.

\begin{tabular}{|c|c|c|c|c|c|c|}
\hline Electrode & Medium & Method & Linear Range & LoD & $E_{\text {Peak }}(V)$ & Reference \\
\hline Cellulose Acetate/GC & $\begin{array}{c}\text { 60:40 } \\
\mathrm{MeOH}-\mathrm{H}_{2} \mathrm{O}\end{array}$ & DPV & $50-180 \mu \mathrm{M}$ & $9.18 \mu \mathrm{M}$ & -1.5 & [15] \\
\hline Vitreous carbon & $\mathrm{EtOH}$ & SWV & & $50 \mathrm{nM}$ & -2.0 & [14] \\
\hline $\mathrm{MnO}_{2} / \mathrm{GC}$ & $\begin{array}{c}60: 40 \\
\mathrm{MeOH}-\mathrm{H}_{2} \mathrm{O}\end{array}$ & Amperometry & $1.1-510 \mu \mathrm{M}$ & $114 \mathrm{nM}$ & -1.45 & [18] \\
\hline Nylon 6,6/MWCNT/Fe ${ }_{3} \mathrm{O}_{4}$ & $\begin{array}{c}\text { 60: } 40 \\
\mathrm{MeOH}-\mathrm{H}_{2} \mathrm{O}\end{array}$ & SWV & $9.9 \mathrm{pM}-5 \mu \mathrm{M}$ & $32 \mathrm{nM}$ & -0.49 & [26] \\
\hline $\mathrm{NiCo}_{2} \mathrm{O}_{4} / \mathrm{GC}$ & $\begin{array}{c}60: 40 \\
\mathrm{MeOH}-\mathrm{H}_{2} \mathrm{O}\end{array}$ & DPV & $10-100 \mu \mathrm{M}$ & $5.9 \mu \mathrm{M}$ & -1.5 & [17] \\
\hline $\mathrm{CuO}-\mathrm{MnO}_{2} / \mathrm{GC}$ & $\begin{array}{c}60: 40 \\
\mathrm{MeOH}-\mathrm{H}_{2} \mathrm{O}\end{array}$ & DPV & $1-700 \mu \mathrm{M}$ & $4.8 \mathrm{nM}$ & -1.5 & [27] \\
\hline Vitreous carbon & DMF & SWV & $40-1000 \mu \mathrm{M}$ & & -1.94 & [11] \\
\hline Polymer/GC & $\mathrm{H}_{2} \mathrm{O}$ & SWV & $0.05-1 \mu \mathrm{M}$ & $30 \mathrm{nM}$ & -0.82 & This work \\
\hline
\end{tabular}

\subsection{Interference Study}

For the interference study, chlorobenzene, 4-chlorobenzaldehyde and 1,3,5-trichlorobenzene were selected for their structural similarity to the target molecule, lindane. Square wave voltammograms were recorded using the modified electrode in $1 \mu \mathrm{M}$ lindane solution in the presence and absence of equal concentration of interfering compounds. The effect of interference was estimated from the relative error by calculating the percentage ratio of the peak current difference in the presence to peak current in the absence of interferent. The relative error obtained in the presence equal molar ratio of chlorobenzene, 4-chlorobenzaldehyde and 1,3,5-trichlorobenzene is $-0.47 \%,-1.5 \%$ and $1.8 \%$, respectively, indicating absence of any interference effect in the determination of lindane. Therefore, the developed sensor has a potential for the selective determination of lindane in the presence of structurally similar compounds.

\subsection{Promising Use in the Field}

The aim of using electrochemical sensors for lindane detection is the promise of device deployment in the fields to avoid manual sampling and centralized sample analyses. Electrochemistry is an emerging technology that has the possibility to identify and quantify different compounds and measure concentrations lower than regulation guidelines requirements. The recent breakthrough in electronics and communication technologies allow the electrochemical method to be applied as a field measuring technique by having a small portable measuring device. This study shows for the first time the simple detection of lindane by polymer-modified sensors with a detection range relevant for field applications.

With the possibility of deploying electronics directly in the field, measurements can be recorded as frequent as relevant. With a measurement time of only $20 \mathrm{~s}$, this technique outcompetes manual sampling, transportation and lengthy analyses of the pesticide content in laboratories. Real-time, online measurements can allow immediate intervention when a site is proven to be contaminated.

The stability of the deposited polymers on the electrode is an important factor when conducting frequent measurements in the field. The modified electrode was stable for repeated use for more than a week after keeping it in an ambient condition. In a previous report, the electrode showed an excellent shelf life stability by maintaining $97.6 \%$ of its initial activity after 25 days of storage in a refrigerator [21].

A real sample from groundwater was spiked with three different concentrations of lindane, $0.1 \mu \mathrm{M}, 0.5 \mu \mathrm{M}$ and $1 \mu \mathrm{M}$. The spiked samples were then tested using the modified electrodes resulting in a linear fit of 0.991 and a slope of 0.739 which does not match the slope presented in Figure 5b. This difference in the slope could be due to the matrix effect of the sample or the change in the ionic strength of the medium when mixed with the ground water $[8,30]$. In addition, the unknown groundwater sample could contain substances that may bind to the polymer and hence cause the shift of the measured signals. 


\section{Conclusions}

Pesticides have been a powerful tool to increase crop yield in the agriculture, however, pesticides are contaminating our environment and polluting our drinking water. To warn consumers about contaminated water, the ultimate goal is to conduct frequent control of the water quality directly in the field. Continuous electrochemical detection of pesticides in the field coupled with an alert system that warns the water suppliers when the pesticide level is exceeding the regulation threshold will make it possible to minimize human exposure to critical pesticides as lindane. Future studies should focus on the development of a full detection system that can be deployed in the field and provide frequent readings on the contaminant level.

Author Contributions: J.S.N. designed and conducted experimental work and data analysis in the reported research, writing and reviewing the paper and conducted the fund raising and project administration. J.M. contributed by supervising the work and reviewing the paper. And A.G. designed and conducted experimental work and data analysis in the reported research in addition to supervising, writing and reviewing the paper. All authors have read and agreed to the published version of the manuscript.

Funding: This research was funded by Fast Track to Innovation program H2020 grant number [820501].

Acknowledgments: The authors would like to thank Senior Researcher Maria Dimaki from the Department of Biotechnology and Biomedicine at the Technical University of Denmark for her great support and feedback during the course of this work. The authors would like to thank Nanna Bild and Ellen Vallentin Christiansen from the Department of Health Technology at Technical University of Denmark for their help in preparing the graphical illustrations. Special thanks for Gevninge Vandværk for providing us with groundwater samples. The authors would like to acknowledge the Fast track to innovation funding program H2020 for supporting this work.

Conflicts of Interest: The authors declare no conflict of interest.

\section{References}

1. Boni, A.C.; Wong, A.; Dutra, R.A.F.; Sotomayor, M.D.P.T. Cobalt phthalocyanine as a biomimetic catalyst in the amperometric quantification of dipyrone using FIA. Talanta 2011, 85, 2067-2073. [CrossRef] [PubMed]

2. Carvalho, F.P. Pesticides, environment, and food safety. Food Energy Secur. 2017, 6, 48-60. [CrossRef]

3. Dalvie, M.A.; Cairncross, E.; Solomon, A.; London, L. Contamination of rural surface and ground water by endosulfan in farming areas of the Western Cape, South Africa. Environ. Health 2003, 2, 1-15. [CrossRef] [PubMed]

4. Parmar, D.; Yadav, S.; Dayal, M.; Johri, A.; Dhawan, A.; Seth, P.K. Effect of lindane on hepatic and brain cytochrome P450s and influence of P450 modulation in lindane induced neurotoxicity. Food Chem. Toxicol. 2003, 41, 1077-1087. [CrossRef]

5. Muir, D.C.; Sverko, E. Analytical methods for PCBs and organochlorine pesticides in environmental monitoring and surveillance: A critical appraisal. Anal. Bioanal. Chem. 2006, 386, 769-789. [CrossRef]

6. Ruzicka, J.; Hansen, E.H. Retro-review of flow-injection analysis. TrAC Trends Anal. Chem. 2008, 27, 390-393. [CrossRef]

7. Girish, K.; Mohammad Kunhi, A.A. Microbial degradation of gamma-hexachlorocyclohexane (lindane). Afr. J. Microbiol. Res. 2013, 7, 1635-1643. [CrossRef]

8. Geto, A.; Noori, J.S.; Mortensen, J.; Svendsen, W.E.; Dimaki, M. Electrochemical determination of bentazone using simple screen-printed carbon electrodes. Environ. Int. 2019, 129, 400-407. [CrossRef]

9. Noori, J.S.; Dimaki, M.; Mortensen, J.; Svendsen, W.E. Detection of Glyphosate in Drinking Water: A Fast and Direct Detection Method without Sample Pretreatment. Sensors 2018, 18, 2961. [CrossRef]

10. Beland, F.A.; Farwell, S.O.; Robocker, A.E.; Geer, R.D. Electrochemical reduction and anaerobic degradation of lindane. J. Agric. Food Chem. 1976, 24, 753-756. [CrossRef]

11. Birkin, P.R.; Evans, A.; Milhano, C.; Montenegro, M.; Pletcher, D. The Mediated Reduction of Lindane in DMF. Electroanalysis 2004, 16, 583-587. [CrossRef]

12. Merz, J.P.; Gamoke, B.C.; Foley, M.P.; Raghavachari, K.; Peters, D.G. Electrochemical reduction of $(1 R, 2 r, 3 S, 4 R, 5 r, 6 S)-$ hexachlorocyclohexane (Lindane) at carbon cathodes in dimethylformamide. J. Electroanal. Chem. 2011, 660, 121-126. [CrossRef]

13. Matsunaga, A.; Yasuhara, A. Dechlorination of polychlorinated organic compounds by electrochemical reduction with naphthalene radical anion as mediator. Chemosphere 2005, 59, 1487-1496. [CrossRef] [PubMed]

14. Martins, P.C.; Medeiros, M.J.; Montenegro, M.I. Electrochemical behaviour of hexachlorocyclo-hexane. Port. Electrochim. Acta 1999, 17, 319-323. [CrossRef] 
15. Kumaravel, A.; Vincent, S.; Chandrasekaran, M. Development of an electroanalytical sensor for $\gamma$-hexachlorocyclohexane based on a cellulose acetate modified glassy carbon electrode. Anal. Methods 2013, 5, 931-938. [CrossRef]

16. Peverly, A.A.; Karty, J.A.; Peters, D.G. Electrochemical reduction of (1R,2r,3S,4R,5r,6S)-hexachlorocyclohexane (Lindane) at silver cathodes in organic and aqueous-organic media. J. Electroanal. Chem. 2013, 692, 66-71. [CrossRef]

17. Prathap, M.A.; Srivastava, R. Electrochemical reduction of lindane $(\gamma-\mathrm{HCH})$ at $\mathrm{NiCo}_{2} \mathrm{O}_{4}$ modified electrode. Electrochim. Acta 2013, 108, 145-152. [CrossRef]

18. Prathap, M.U.A.; Sun, S.; Xu, Z.J. An electrochemical sensor highly selective for lindane determination: A comparative study using three different $\alpha-\mathrm{MnO} 2$ nanostructures. RSC Adv. 2016, 6, 22973-22979. [CrossRef]

19. Prathap, M.U.A.; Sun, S.; Wei, C.; Xu, Z.J. A novel non-enzymatic lindane sensor based on $\mathrm{CuO}_{-} \mathrm{MnO}_{2}$ hierarchical nanomicrostructures for enhanced sensitivity. Chem. Commun. 2015, 51, 4376-4379. [CrossRef]

20. Geto, A.; Brett, C.M. Electrochemical synthesis, characterisation and comparative study of new conducting polymers from amino-substituted naphthalene sulfonic acids. J. Solid State Electrochem. 2016, 20, 2969-2979. [CrossRef]

21. Geto, A.; Amare, M.; Tessema, M.; Admassie, S. Voltammetric Determination of Nicotine at Poly(4-Amino-3-Hydroxynaphthalene Sulfonic Acid)-Modified Glassy Carbon Electrode. Electroanalysis 2012, 24, 659-665. [CrossRef]

22. Tefera, M.; Geto, A.; Tessema, M.; Admassie, S. Simultaneous determination of caffeine and paracetamol by square wave voltammetry at poly(4-amino-3-hydroxynaphthalene sulfonic acid)-modified glassy carbon electrode. Food Chem. 2016, 210, 156-162. [CrossRef]

23. Geto, A.; Tessema, M.; Admassie, S. Determination of histamine in fish muscle at multi-walled carbon nanotubes coated conducting polymer modified glassy carbon electrode. Synth. Met. 2014, 191, 135-140. [CrossRef]

24. Farwell, S.O.; Beland, F.A.; Geer, R.D. Interrupted-sweep voltammetry for the identification of polychlorinated biphenyls and naphthalenes. Anal. Chem. 1975, 47, 895-903. [CrossRef]

25. Bard, A.J.; Faulkner, L.R. Electrochemical Methods, Fundamentals and Applications, 2nd ed.; Wiley: New York, NY, USA, 2001.

26. Kalaiyarasi, J.; Meenakshi, S.; Gopinath, S.C.B.; Pandian, K. Mediator-free simultaneous determination of acetaminophen and caffeine using a glassy carbon electrode modified with a nanotubular clay. Microchim. Acta 2017, 184, 4485-4494. [CrossRef]

27. Shrivastava, A.; Gupta, V.B. Methods for the determination of limit of detection and limit of quantitation of the analytical methods. Chron. Young Sci. 2011, 2, 21-25. [CrossRef]

28. Noori, J.S.; Mortensen, J.; Geto, A. Recent Development on the Electrochemical Detection of Selected Pesticides: A Focused Review. Sensors 2020, 20, 2221. [CrossRef]

29. Fayemi, O.E.; Adekunle, A.S.; Ebenso, E.E. A Sensor for the Determination of Lindane Using PANI/Zn, Fe(III) Oxides and Nylon 6,6/MWCNT/Zn, Fe(III) Oxides Nanofibers Modified Glassy Carbon Electrode. J. Nanomater. 2016, 2016, 4049730. [CrossRef]

30. Lezi, N.; Economou, A. Voltammetric Determination of Neonicotinoid Pesticides at Disposable Screen-Printed Sensors Featuring a Sputtered Bismuth Electrode. Electroanalysis 2015, 27, 2313-2321. [CrossRef] 